\section{Relación entre calidad de vida y proceso de cicatrización en heridas crónicas complicadas} Relationship between life quality
and cicatrization process in.
complicated chronicle wounds

\author{
Estrella Perdomo Pérez ${ }^{1, *}$ \\ Javier Soldevilla Agreda ${ }^{2}$ \\ Francisco Pedro García Fernández ${ }^{3}$ \\ 1. Enfermera. Máster Universitario en gestión integral e investigación de las heridas \\ crónicas por la Universidad de Cantabria. Máster en UPP y Heridas crónicas por la \\ Universidad de Cantabria. Responsable de la Unidad de Heridas Complicadas de AP \\ de GC. Servicio Canario de la Salud. Miembro del Comité Director del GNEAUPP. \\ 2. Enfermero. Doctor por la Universidad de Santiago. C.S. Ausejo. Servicio Riojano \\ de la Salud. Profesor de Enfermería Geriátrica. Escuela de Enfermería de Logroño. \\ Director del GNEAUPP. \\ 3. Enfermero. Doctor por la Universidad de Jaén. Profesor del Departamento de Enfermería \\ de la Facultad de Ciencias de la Salud. Universidad de Jaén. Miembro de la Cátedra de \\ Estudios Avanzados en Heridas UJA-GNEAUPP. Miembro Comité Director del GNEAUPP.
}

*Autor para correspondencia.

Correo electrónico: eperdomo1958@gmail.com (Estrella Perdomo Pérez).

Recibido el 21 de febrero de 2020; aceptado el 9 de marzo de 2020.

\section{RESUMEN}

Introducción: Las heridas crónicas afectan a la calidad de vida relacionada con la salud (CVRS) de las personas que las padecen, especialmente cuando son lesiones de difícil cicatrización, que se alargan en el proceso de curación. Objetivo: Determinar la CVRS de los pacientes con heridas crónicas y analizar cómo la evolución clínica de la herida influye en las distintas dimensiones de la CVRS, mediante

la aplicación del instrumento Cardiff Wound Impact Schedule (CWIS). Metodología: Estudio observacional de medidas repetidas de una cohorte de pacientes con heridas crónicas complicadas. Se analizó la situación basal de la CVRS mediante el CWIS y se buscó la relación existente entre la cicatrización de la lesión medida por RESVESH 2.0 y la CVRS medida por la puntuación CWIS. El estudio se desarrolló en la Unidad de Heridas Complicadas (de Atención Primaria de Gran Canaria y del Hospital Dr Negrín). Se incluyó a pacientes con heridas de etiología diversa que reunían las características que las definían como crónico/complejas. Se excluyeron heridas agudas, personas que no tenían capacidad para otorgar su consentimiento o no entendían el idioma castellano. El tamaño se fijó en 65 pacientes, que fueron seleccionados mediante un muestreo accidental o de conveniencia desde la fecha de inicio del estudio hasta completar el tamaño muestral. Resultados: Al inicio del estudio, la calidad de vida medida mediante CWIS está por debajo del $50 \%$ de la puntuación máxima (113 sobre 245), es decir tienen una calidad de vida baja, que mejora notablemente conforme lo hace la lesión de los pacientes y que al final del estudio llega al 78\%. También se analizaron las correlaciones entre inicio y mes de inicio y final, segmentando la muestra por las diferentes variables, sexo, cicatrización completa o no, tipo de lesión, lesión recidivante o forma de convivencia, y en ningún caso se hallaron relaciones estadísticamente significativas ( $p>0,05$ en todos los casos) y solo existió una correlación entre la mejora de la lesión al mes medida por RESVECH y la subescala calidad de vida global; el resto no tuvo significación estadística. Conclusiones: Los resultados mostraron que las heridas crónicas habían comprometido la calidad de vida, y el dominio de "bienestar" era el más afectado, especialmente cuando se asociaba con factores clínicos. Entre las condiciones clínicas asociadas

con peor calidad de vida, se destacó la duración, el tipo de herida, la profundidad, el aspecto, exudado, olor y el dolor. Al inicio del estudio,

\section{SUMMARY}

Introduction: Chronic wounds affect the health-related quality of life (HRQL) of people who suffer them, especially when these are difficult-to-heal injuries, which lengthen in the healing process. Aims: Determine the HRQL of patients with chronic wounds and analyze how the clinical evolution of the wound influences the different dimensions of HRQL, by applying the Cardiff Wound Impact Schedule (CWIS) instrument. Methodology: Observational repeated measures study of a cohort of patients with complicated chronic wounds (HCC). The baseline situation of HRQL was analyzed using the CWIS and the existing relationship of lesion healing measured by RESVESH 2.0 and HRQL measured by the CWIS score were searched. The study was carried out in the Complicated Wounds Unit (Primary Care of Gran Canaria and Hospital Dr Negrin). Patients with wounds of diverse etiology that met the characteristics that defined them as chronic / complex were included. Acute injuries were excluded for people who did not have the capacity to give their consent or who did not understand the Spanish language. The size has been set at 65 patients who were selected by accidental or convenience sampling from the start date of the study to complete the sample size. Results: At the start of the study, the quality of life measured by CWIS is below $50 \%$ of the maximum score (113 out of 245 ), that is, they have a low quality of life, improving significantly as the injury of the patients improves and that at the end of the study reaches $78 \%$. The correlations between start and month and start and end were also analyzed, segmenting the sample by the different variables, sex, complete healing or non-type of injury, recurrent injury or type of coexistence, and in no case were statistically significant relationships found ( $p>0.05$ in all cases) and there was only a correlation between improvement of the lesion per month as measured by RESVECH and global quality of life subscale, the rest did not have statistical significance. Conclusions: The results showed that chronic wounds had compromised quality of life and the "well-being" domain was the most affected, especially when it was associated with clinical factors and among the clinical conditions associated with poorer quality of life, duration, wound type, depth, exudate, odor, and pain. At the start of the study, 
cuando las lesiones no habían recibido un tratamiento óptimo, se halló que la calidad de vida de los pacientes fue baja, pero mejoró notablemente al final trabajo.

PALABRAS CLAVE: Calidad de vida, cicatrización de lesiones, heridas complicadas. when the lesions had not received optimal treatment, it was found that the quality of life of the patients was low, improving markedly at the end of the work.

KEYWORDS: Quality of life, wound healing, complicated wounds.

\section{$\vee$ INTRODUCCIÓN}

Una herida es una pérdida de continuidad de la piel o mucosa producida por algún agente físico o químico, que cursa con una serie de signos y síntomas, tales como separación de bordes de la piel, dolor, inflamación, hemorragia, etc. ${ }^{1}$.

La cicatrización comienza en el mismo momento en que se produce la lesión. Es un proceso biológico, con reacciones bioquímicas y mitóticas celulares, que persigue el cierre de la herida, ya sea por primera o por segunda intención ${ }^{2}$. Es un proceso continuo y simultáneo, donde las diferentes fases se solapan para conseguir el reemplazo de la piel por un tejido sustituto.

En las lesiones etiquetadas como heridas crónicas: úlceras vasculares, úlceras diabéticas, procesos neoplásicos, o iatrogénicas como las lesiones por presión, suele haber un componente endógeno principal, ya sea de origen metabólico o alguna enfermedad de base que produce un retraso en el tiempo de curación y una ausencia de crecimiento de los tejidos. Estas lesiones suponen una sensible disminución en la calidad de vida en los pacientes que las portan ${ }^{3}$.

La calidad de vida relacionada con la salud (CVRS) se entiende como "el valor asignado a la duración de la vida en función de la percepción de limitaciones físicas, psicológicas, sociales y de disminución de oportunidades a causa de la enfermedad, sus secuelas, el tratamiento y/o las políticas de salud"4.

Esta definición es muy útil para describir el impacto que tiene la enfermedad en los pacientes, y también para evaluar si los tratamientos que reciben están beneficiando o no su calidad de vida.

La CVRS puede estar influida por la presencia de heridas, la cuales pueden estar acompañadas de cambios en la vida diaria, sufrimiento, dolor, limitaciones, dependencia, alteración de la autoestima, temor, angustia y aislamiento, entre otros. Algunas investigaciones han reportado asociación entre CVRS y cicatrización: inflamación y tamaño de la herida; dolor; impedimentos físicos como consecuencia de las heridas, y amputación mayor o menor ${ }^{5}$. Asimismo, existe una relación directa entre la gravedad de la herida y la pérdida de CVRS ${ }^{6}$. Por tanto, es necesario valorar el impacto en la CVRS en los pacientes con heridas para optimizar los cuidados que se les proporcionan.

Según Santos et al., para medir la CVRS de pacientes con heridas crónicas, los instrumentos deben tener en cuenta tanto aspectos propios de estas patologías como la percepción que tienen de ellos mismos, los aspectos sociales y los emocionales. Existen instrumentos de tipo genérico para la medición de la CVRS en pacientes con heridas crónicas ${ }^{7}$.

Existen algunos instrumentos validados para medir la CVRS de las personas con heridas, como el Cardiff Wound Impact Schedule (CWIS). Se trata de un cuestionario de 28 preguntas que valora síntomas físicos y de la vida diaria, la vida social y el bienestar. Su validez y fiabilidad está confirmada con pruebas psicométricas positivas. Este instrumento ha sido validado y traducido en diferentes países europeos, latinoamericanos y al inglés de EE. UU. ${ }^{8}$.
Por este motivo, creemos necesario desarrollar un estudio que determine cómo influye la evolución de las lesiones en la CVRS y si los instrumentos utilizados y validados en otros contextos son también válidos en nuestro país.

\section{Y OBJETIVOS}

- Evaluar las dimensiones del CVRS según el CWIS en la población con heridas crónicas complejas.

- Estudiar el proceso de evolución clínica hacia la cicatrización de las heridas crónicas, previa aplicación de un instrumento de preparación del lecho de la herida (DOMINATE-WOUND) ${ }^{9}$ y la utilización de la herramienta RESVESH 2.0 $0^{10}$.

- Establecer la relación entre la cicatrización de la lesión medida por puntuación RESVESH 2.0 y la CVRS medida por la puntuación CWIS.

\section{METODOLOGÍA}

\section{Tipo de estudio}

Se planteó un estudio observacional prospectivo longitudinal de medidas repetidas de una cohorte de pacientes con heridas crónicas complicadas (HCC).

Se analizó la situación basal de la CVRS de los pacientes mediante el CWIS cuando fueron incluidos en el estudio y derivados a las unidades participantes. Posteriormente, se preparó el lecho de la herida mediante la aplicación de la herramienta Dominate-Wound ${ }^{9}$ y la utilización de la herramienta RESVESH 2.0, y se buscó la relación existente entre la cicatrización de la lesión medida por puntuación RESVESH 2.0 y la CVRS medida por la puntuación CWIS.

\section{Ámbito de estudio}

El estudio se desarrolló en la Unidad de Heridas Complicadas perteneciente al ámbito de Atención Primaria del Área de Gran Canaria y la Unidad de Heridas Complicadas de Cirugía Vascular del Hospital Dr. Negrín que atiende a usuarios de la zona norte de Gran Canaria, ambas unidades dependientes del Servicio Canario de la Salud.

\section{Unidad de estudio}

- Población: pacientes portadores de heridas complejas atendidos en la Unidad de Heridas complicadas de Atención Primaria de GC y en la del Servicio de Cirugía Vascular del Hospital Dr. Negrín de Las Palmas de Gran Canaria.

- Muestra: se seleccionaron, del total de la población, aquellos pacientes que fueron atendidos durante el periodo de estudio hasta completar el tamaño muestral. 
- Criterios de inclusión: pacientes con HCC de etiología diversa que reunieron las características que las definían como tales.

- Criterios de exclusión: pacientes con heridas agudas, que no tenían capacidad para otorgar su consentimiento ni tutor que pudiera hacerlo o que no entendían el idioma castellano.

- Tamaño de la muestra: para conseguir una potencia del $80,00 \%$ para detectar diferencias en el contraste de la hipótesis mediante una prueba $t$ de Student bilateral para dos muestras relacionadas, teniendo en cuenta que el nivel de significación es del 5,00\% y asumiendo que la media de la puntuación RESVECH al inicio del estudio era de 20 puntos, la media al mes, de 18, y la desviación típica de la variable diferencia de 5,50 puntos, se estimó necesario incluir 65 pacientes para medidas repetidas en el estudio.

- Método de selección de la muestra: se realizó un muestreo de tipo intencional o de conveniencia desde la fecha de inicio del estudio hasta completar el tamaño muestral.

\section{Variables analizadas}

- Variables sociodemográficas: edad, sexo, nivel de estudios, patologías asociadas según la clasificación CIE-9, centro de procedencia y profesional que deriva.

- Variables relacionadas con las heridas: tipo de herida, lesión nueva o recidivante, tiempo de evolución, valoración inicial y final de la lesión y cicatrización completa de la lesión.

- Variables relacionadas con la calidad de vida: valoración inicial y final de la CVRS.

\section{Métodos e instrumentos para la recogida de datos}

Los datos fueron incluidos en un cuaderno diseńado "ad hoc" por los investigadores con las variables del estudio descritas anteriormente y los datos relativos al proceso de cicatrización mediante Dominate-Wounds. Como instrumentos de recogida de datos se utilizaron:

- Cardiff Wound Impact Schedule en su versión española (CWIS), que es un instrumento específico de medición de CVRS para heridas crónicas. Consta de 47 preguntas relativas a los datos personales y CVRS, además de las dimensiones respecto a los síntomas físicos y de vida diaria, vida social y bienestar, con una puntuación que oscila entre 39 (peor calidad de vida) y 245 (máximo nivel de calidad de vida). Este cuestionario tiene pruebas psicométricas positivas que confirman su validez y fiabilidad. Además, ha sido traducido y validado en Portugal, Alemania, Francia, México ${ }^{11,12}$, y en castellano en una tesis pendiente de publicar ${ }^{13}$.

- RESVECH: es un instrumento de monitorización clínica y medida de la cicatrización en heridas crónicas. Se compone de 9 variables, cada una de ellas con diferentes subcategorías de calificación, las cuales se asignan en el momento de la valoración por el evaluador (dimensiones de la úlcera, profundidad/tejidos afectados, bordes, presencia de maceración perilesional, presencia de tunelizaciones, tipo de tejido en el lecho de la herida, exudado, infección/inflamación y presencia de dolor. La puntuación de RESVECH oscila entre 0 , lesión curada y 35 peor opción de la lesión ${ }^{10}$.

\section{Análisis de los datos}

Se ha realizado un análisis secuencial. Inicialmente, en el análisis descriptivo, las variables cuantitativas se presentan como medias y valores máximos y mínimos. Las variables cuantitativas se presentan como frecuencias y porcentajes. Para evaluar las dimensiones de CVRS según el CWIS en la población con HCC se calcularon las medias de puntuación con su desviación estándar en cada uno de los subgrupos que constituyen el cuestionario y la puntuación global del mismo en los diferentes momentos del estudio.
La diferencia de medias fue medida por la t de Student para muestras relacionadas para variables dicotómicas y ANOVA de medidas repetidas para variables policotómicas, en función de los criterios de normalidad que se obtuvieron al realizar el contraste mediante la prueba de Kolmorov-Smirnov.

Para comparar la influencia de las variables sociodemográficas en la cicatrización de las heridas y la calidad de vida se utilizó la prueba de la $t$ de Student para muestras independientes y el test de ANOVA en las policotómicas tras comprobar la normalidad de las mismas.

Para determinar el proceso de evolución clínica hacia la cicatrización de las HC se calculó el porcentaje de cicatrización de las lesiones mediante la fórmula:

\section{(RESVECH inicial o medio - RESVECH final) *100/RESVECH inicial}

Para determinar el proceso de evolución de la calidad de vida se calculó el porcentaje de esta mediante la fórmula:

\section{(CWIS final o medio - CWIS inicial) *100/RESVECH inicial}

Para determinar la relación entre la cicatrización de la lesión medida por puntuación RESVESH 2.0 y CVRS medida por la puntuación CWIS se midió la R de Pearson para la correlación entre dos variables cuantitativas (mediante pruebas paramétricas).

En todos los casos se trabajó con un nivel de confianza del $95 \%$, por lo que se estima significativo un valor $p<0,05$.

\section{Aspectos éticos}

Se garantizó la confidencialidad de los datos: no se utilizaron sus nombres, ni en el informe final, ni durante la codificación, análisis y difusión de los datos según la Ley Orgánica 3/2018, de 5 de diciembre, de Protección de Datos Personales y garantía de los derechos digitales. (Ley Orgánica que tiene por objeto garantizar y proteger, en lo que concierne al tratamiento de los datos personales, las libertades públicas y los derechos fundamentales de las personas físicas, y especialmente de su honor e intimidad personal y familiar.) Como se trata de una práctica habitual, simplemente a los pacientes se les solicitó su consentimiento informado para poder incluir su caso dentro de este estudio, sin menoscabo de ningún derecho, sabiendo que podían ejercer sus derechos de acceso, rectificación, cancelación u omisión en cualquier momento.

\section{$\searrow$ RESULTADOS}

\section{Perfil de los pacientes incluidos}

La muestra incluyó 65 pacientes, de los que el $60 \%$ eran hombres con una media de 60,09 ańos (34-85 ańos). El 80\% procedía de atención primaria. El $40 \%$ tenía estudios primarios y un $26 \%$, estudios secundarios. Prácticamente el $50 \%$ eran de centros urbanos y rurales. La derivación procedió mayoritariamente de las enfermeras, seguida por la derivación médica. Las patologías según CIE-9 asociadas a los procesos estudiados en función de la frecuencia fueron: insuficiencia venosa, diabetes, diabetes e hipertensión arterial, pluripatologías, hipertensión arterial pura, UPP-LRD y síndrome $\mathrm{X}$ (síndrome dismetabólico) con insuficiencia venosa. El 90\% de los pacientes incluidos en el estudio refirieron vivir acompañados. Sobre la frecuencia de ver a su familia, la mayoría $(81,50 \%)$ refirió verla a diario, dato concordante con el elevado número de pacientes que vivían acompañados. Uno de cada diez lo hace una vez a la semana, y en porcentajes menores a una vez al mes $(3,10 \%)$ o menos de una vez al mes $(4,60 \%)$. 


\section{Relacionados con las heridas}

Como podemos ver en la figura 1, el mayor porcentaje de las heridas incluidas en el estudio fueron lesiones de origen venoso, seguidas de lesiones diabéticas. La media del tiempo de evolución de las lesiones fue de 9,46 meses, con un tiempo mínimo de 0 meses y un máximo de 35 meses. Casi todas las lesiones $(83,1 \%)$ fueron recidivantes, lo que puede tener relación con el tipo de lesión más frecuente recogida en el estudio (úlceras venosas). Sobre el porcentaje de cicatrización: casi 9 de cada 10 lesiones cicatrizaron completamente durante el estudio y el $100 \%$ de las lesiones estudiadas presentaron una evolución favorable a la conclusión del trabajo.

\section{Sobre la cicatrización de las lesiones}

En la tabla 1 se presenta la evolución de la puntuación RESVECH en los distintos momentos del estudio. Las heridas al inicio del estudio son lesiones importantes con puntuaciones elevadas, lo que indica un manejo complejo de estas. Al mes han evolucionado con una reducción de su tamaño que supera el $30 \%$, y al final del estudio las puntuaciones indican que prácticamente están cicatrizadas.

Asimismo, se realizaron comparaciones entre las puntuaciones de RESVECH al inicio, mes y final en función de las variables sociodemográficas analizadas, sexo, nivel de estudios, tipo de lesión, si la lesión es recidivante o no y tipo de convivencia, no teniendo ninguna de ellas diferencias significativas $(p>0,05)$.

De todas las lesiones al inicio, las lesiones diabéticas son las que tienen peor situación clínica medida por RESVECH, seguidas de las arteriales y venosas (tabla 2), y las únicas que muestran diferencias significativas al inicio y al final.

La evolución de las lesiones fue muy similar independientemente de que esta fuera nueva o recidivante, por lo que esta variable no influyó en las mismas. Sobre el hecho de vivir solo, esta situación hace que al iniciarse las lesiones estas tengan peor puntuación en la escala RESVECH, es decir, son más graves (23,33/35 frente a 17,15/35 en RESVECH; $p=0,01)$ y estas diferencias son significativas, lo que se mantiene al mes $(p=0,03)$ y al final $(p=0,01)$. Esto significa que las heridas de los pacientes que tienen a alguien que los cuide cicatrizan mucho más y por eso las diferencias de las puntuaciones de RESVECH son mayores, por lo que el hecho de vivir solo podría ser considerado como factor de riesgo en la cicatrización.

\section{Datos relativos a la calidad de vida}

$\mathrm{Al}$ inicio del estudio, la calidad de vida medida mediante CWIS está por debajo del $50 \%$ de la puntuación máxima (113 sobre 245), es decir,

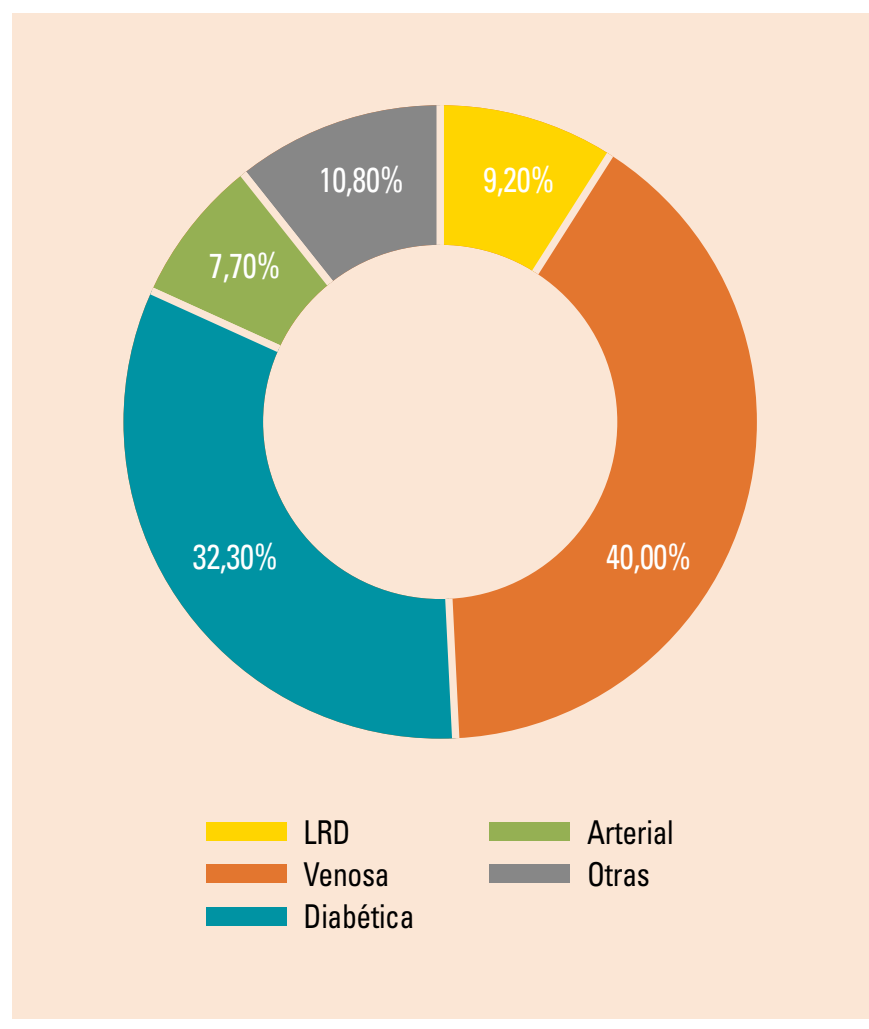

Figura 1. Tipos de lesiones.

LRD: lesiones relacionadas con la dependencia.

Tabla 1. Evolución de RESVECH en los distintos momentos (inicio, mes y final) y porcentaje de reducción

\begin{tabular}{|c|c|c|c|c|}
\hline & $\begin{array}{c}\text { RESVECH } \\
\text { medio }\end{array}$ & $\begin{array}{c}\text { Reducción } \\
\text { (en puntos) }\end{array}$ & $\begin{array}{c}\text { Reducción } \\
(\%)^{\mathrm{a}}\end{array}$ & VALOR R $^{\mathbf{b}}$ \\
\hline Inicio-Mes & $17,72-12,09$ & 5,63 & $31,77 \%$ & $\begin{array}{c}0,805 \\
(p<0,0001)\end{array}$ \\
\hline Mes-Final & $12,09-01,37$ & 10,72 & $60,49 \%$ & $\begin{array}{c}0,706 \\
(p<0,0001)\end{array}$ \\
\hline Inicio-Final & $17,72-01,37$ & 16,75 & $94,53 \%$ & $\begin{array}{c}0,941 \\
(p<0,0001)\end{array}$ \\
\hline
\end{tabular}

`Calculado sobre la puntuación media al inicio de las lesiones.

balor R medido mediante el coeficiente de correlación y nivel de significación estadística.

Tabla 2. Evolución de RESVECH en función del tipo de lesión

\begin{tabular}{|l|c|c|c|c|c|c|}
\hline \multicolumn{1}{|c|}{ Tipo de lesión } & N & RESVECH inicial & RESVECH mes & REVESCH final & Difer. Inicio-Mes & Difer. Inicio-Final \\
\hline Otras & 7 & 13,57 & 9,33 & 1,00 & 4,24 & 12,57 \\
\hline LRD & 6 & 14,67 & 10,31 & 1,29 & 4,36 & 13,38 \\
\hline Venosa & 26 & 17,00 & 11,29 & 1,40 & 5,71 & 15,60 \\
\hline Arterial & 5 & 17,20 & 13,60 & 1,42 & 3,6 & 15,78 \\
\hline Diabética & 21 & 21,00 & 15,00 & 1,71 & 6,0 & $\mathbf{1 9 , 2 9}$ \\
\hline
\end{tabular}

aDiferencias significativas según la prueba de ANOVA.

LRD: Lesiones relacionadas con la dependencia. 
tienen una calidad de vida baja, que mejora notablemente conforme lo hace la lesión de los pacientes y que al final del estudio llega al 78\% de la puntuación máxima del cuestionario (tabla 3). Estas mejoras del nivel están altamente relacionadas entre el inicio y el mes $(R>0,7)$, medianamente relacionadas entre mes y final, y con una correlación baja inicio y final, pero en todos los casos las diferencias son estadísticamente significativas.

Todas las puntuaciones de cada subescala están en torno o por debajo del 50\% de dicha subescala, siendo las de bienestar y calidad de vida global las que en proporción reciben la puntuación más baja (tabla 4).

Posteriormente, hay una mejoría generalizada en todos los ítems de las subescalas, y vemos que conforme avanza la cicatrización de las lesiones hace que mejoren todos los aspectos de la calidad de vida, especialmente el de bienestar, que era, como hemos mencionado, el que más bajo partía y el que dobla su puntuación al final del proceso. También queremos destacar las puntuaciones de las subescalas calidad de vida global y satisfacción con la calidad de vida, que son las más directas al puntuar de $0 \mathrm{a}$ 10 y que pasan de menos de un 5 a casi 7,5 puntos de la escala.

Se constató que ni el sexo, ni el nivel de estudios, ni el tipo de lesión influyó en la puntuación global de la calidad de vida en ninguno de los tres momentos $(p>0,05)$.

Tabla 3. Evolución de CWIS en los distintos momentos (inicio, mes y final) y porcentaje de reducción

\begin{tabular}{|c|c|c|c|c|}
\hline & CW/S medio & $\begin{array}{c}\text { Mejora } \\
\text { (en puntos) }\end{array}$ & Mejora (\%) & Valor R \\
\hline Inicio-Mes & $137,80-113,71$ & 24,09 & $21,18 \%$ & $\begin{array}{c}0,861 \\
(p<0,0001)\end{array}$ \\
\hline Mes-Final & $191,75-137,80$ & 53,95 & $47,44 \%$ & $\begin{array}{c}0,597 \\
(p<0,0001)\end{array}$ \\
\hline Inicio-Final & $191,75-113,71$ & 78,04 & $68,63 \%$ & $\begin{array}{c}0,390 \\
(p=0,001)\end{array}$ \\
\hline
\end{tabular}

aCalculado sobre la puntuación media al inicio del CWIS.

valor R medido mediante el coeficiente de correlación y nivel de significación estadística.

Tabla 4. Evolución de las subescalas de CWIS en los distintos momentos (inicio, mes y final)

\begin{tabular}{|c|c|c|c|c|}
\hline Subescala & Inicio & Mes & Final & $\begin{array}{l}\text { Rango de } \\
\text { puntuación }\end{array}$ \\
\hline Vida social (estrés) & 17,51 & 18,74 & 25,11 & $5-35$ \\
\hline $\begin{array}{l}\text { Vida social } \\
\text { (problemas) }\end{array}$ & 18,20 & 20,22 & 26,86 & $5-35$ \\
\hline Bienestar & 13,26 & 14,43 & 25,26 & $5-35$ \\
\hline $\begin{array}{l}\text { Síntomas físicos } \\
\text { (estrés) }\end{array}$ & 28,45 & 33,89 & 49,92 & $12-60$ \\
\hline $\begin{array}{l}\text { Síntoma físicos } \\
\text { (problemas) }\end{array}$ & 27,00 & 34,22 & 49,85 & $12-60$ \\
\hline $\begin{array}{l}\text { Calidad de vida } \\
\text { global }\end{array}$ & 4,51 & 5,80 & 7,37 & $0-10$ \\
\hline $\begin{array}{l}\text { Satisfacción con la } \\
\text { calidad de vida }\end{array}$ & 4,78 & 5,72 & 7,34 & $0-10$ \\
\hline
\end{tabular}

Inicialmente, la calidad de vida es mejor en los pacientes con lesión recidivante que ya conocen la situación de las lesiones $(p=0,04)$. Las lesiones nuevas que partieron de una peor calidad de vida (109/234 frente a los 135/235 de las recidivantes) presentaron una mejora muy notable en cuanto a la misma al mes y al final del estudio, llegando a superar la puntuación del CWIS (195/235 de la lesión nueva frente a los $185 / 235$ de la recidivante) $(p=0,02)$.

$\mathrm{Al}$ igual que con el estado de las lesiones, existieron diferencias estadísticamente significativas en cuanto a la calidad de vida al inicio del estudio de los pacientes en función de si vivían solos o acompañados, teniendo peor calidad de vida los que vivían solos $(p<0,0001)$, manteniéndose las diferencias al mes $(p=0,006)$ y al final del estudio $(p=0,02)$.

\section{Relación entre estado de la lesión y la calidad de vida}

Se encontró una correlación entre el estado de la lesión y la calidad de vida: entre las diferencias al inicio y al mes del estado de la lesión $(p=0,25)$, dicha correlación fue débil $(\mathrm{R}<0,3)$; sin embargo, esta correlación desapareció al medir las diferencias al inicio y al final del estudio, ya que la mayoría de las lesiones cicatrizaron.

También se analizaron las correlaciones entre inicio y mes desde el inicio y final, segmentando la muestra por las diferentes variables: sexo, cicatrización completa o no, tipo de lesión, lesión recidivante o forma de convivencia, y en ningún caso se encontraron relaciones estadísticamente significativas $(p>0,05)$; solo existió una correlación entre la mejora de la lesión al mes medida por RESVECH y la subescala calidad de vida global $(p=0,002)$; el resto no tiene significación estadística. Para hacer un análisis más detallado, se recodificó la variable RESVECH en una variable dicotómica (poca mejoría o mucha mejoría) en función de que la reducción de la cicatrización de la lesión estuviera por debajo o por encima del percentil 50 (mediana) de la disminución del tamaño de la herida, comparando las diferencias con el mes de CWIS y con cada una de las subescalas. Nuevamente, solo la subescala calidad de vida se relaciona de forma estadísticamente significativa con la mejoría de la lesión $(p=0,005)$. Se realizó el mismo procedimiento, recodificando la variable RESVECH en una variable dicotómica en función de que la reducción de la cicatrización de la lesión estuviera por debajo o por encima del percentil 50 (mediana) de la disminución del tamaño de la herida medido por RESVECH, comparando las diferencias inicio-final de CWIS y con cada una de las subescalas, y de nuevo ninguna subescala guardó relación estadísticamente significativa con la mejoría de la lesión $(p>0,05)$.

\section{У DISCUSIÓN}

El presente estudio halló un perfil de paciente relativamente joven (en torno a 60 años) con una evolución de casi un año de la lesión, similar a lo encontrado por Panier y Rabe ${ }^{14}$ y algo mejor que lo encontrado por otros autores ${ }^{15,16}$, con un nivel de estudios primarios, datos que concuerdan también con los presentados por otros autores ${ }^{17,18}$.

Casi todas las lesiones estudiadas fueron recidivantes, con tasas superiores a las registradas por otros autores ${ }^{19-20}$, siendo las úlceras venosas las más importantes, lo que básicamente es apoyado por casi todos los trabajos consultados ${ }^{21-22}$.

La cicatrización completa se registró en torno al $90 \%$ de las lesiones durante los 12 meses que duró el estudio. Frente a ello, fue difícil encontrar trabajos que constataran intervalos de tiempo para cicatrización; no obstante, se halló evidencia de úlceras cicatrizadas en 6 meses en un $86 \%$ de las lesiones ${ }^{16}$. 
De todas las lesiones al inicio, las lesiones diabéticas son las que tienen peor situación clínica, aunque luego son las que presentan mejor evolución entre inicio y final del proceso, lo que concuerda con las recogidas por Zimny et al. ${ }^{23}$.

Hemos mostrado la importancia de vivir solo en la situación inicial y en la evolución de las lesiones, que es peor que la de los pacientes que tienen un cuidador, lo que concuerda con las afirmaciones planteadas por Fernández Monteguín: "El aislamiento social, con ausencia de contactos con vecinos, amigos, familiares y la comunidad, repercute negativamente en la severidad y evolución de las lesiones de estos enfermos" 24 .

Sin embargo, en otros trabajos se recoge que los pacientes que viven solos tienen mejor calidad de vida relacionada con la salud que aquellos que dependen de los demás ${ }^{25}$. Al inicio del estudio, la calidad de vida medida mediante CWIS está por debajo del $50 \%$ de la puntuación máxima, es decir, tienen una calidad de vida baja, dato algo inferior al encontrado en otros trabajos como el de Kaps y Santamaría ${ }^{26}$.

La calidad de vida relacionada con la salud fue subóptima (6/10) de acuerdo con el Programa de Impacto de Heridas de Cardiff, especialmente la de bienestar y calidad de vida global, que en proporción reciben la puntuación más baja. Otros estudios también las reflejan como las más afectadas ${ }^{27,28}$. Conforme avanzó la cicatrización mejoraron todos los aspectos de la calidad de vida. Otros trabajos encontrados recogen que, tras intervenciones en personas con lesiones, al disminuir los síntomas y aumentar la cicatrización se obtiene como respuesta una mejoría en la percepción de CVRS $^{29,30}$. No hemos hallado diferencias en cuanto a las principales variables sociodemográficas, lo que en cuanto al sexo es apoyado por otros estudios ${ }^{25}$, aunque otros autores sí encontraron diferencias en el nivel de escolaridad ${ }^{31-33}$ y calidad de vida, resultado que concuerda con el encontrado por González-Consuegra y Verdú, donde los pacientes manifiestan mejoría en su CVRS a medida que su estado mejora $^{32}$.

No obstante, el CWIS, la subescala calidad de vida global, parece ser suficiente para medir la calidad sin que el resto del cuestionario aporte nada nuevo. Esto también es recogido por Saraiva et al. ${ }^{33}$.

\section{$\searrow$ CONCLUSIONES}

- El perfil del paciente con herida crónica es un adulto joven con lesión venosa de casi un año de evolución.

- Las lesiones diabéticas son las que tuvieron peor situación clínica medida por RESVECH, seguidas de las arteriales y venosas.

- Las lesiones en pacientes que vivían solos fueron, al inicio del estudio, más graves.

- Al inicio del estudio, se encontró que la calidad de vida de los pacientes fue baja, pero mejoró notablemente conforme avanzó la cicatrización.

- Ni el sexo, ni el nivel de estudios, ni el tipo de lesión influyó en la CVRS.

- Los pacientes con lesiones recidivantes presentaron mejor calidad de vida al inicio, igualándose posteriormente al mes y al final del estudio. Las lesiones nuevas que partían de peor calidad de vida tuvieron una mejora muy notable en cuanto a la misma, al mes y al final del estudio, diferencias que fueron significativas con respecto a las lesiones recidivantes.

- Los pacientes que vivían solos presentaron una peor calidad de vida, con una diferencia importante, frente a los que vivían acompańados.

- Existe una correlación entre el estado de la lesión y la calidad de vida, entre las diferencias al inicio y al mes del estado de la lesión.

- La subescala calidad de vida es suficientemente sensible para medir la CVRS y la relación con la cicatrización sin que el resto del cuestionario de CWIS aporte nada a la misma

\section{Agradecimientos}

A los compańeros que participaron en la recogida de datos del presente trabajo, a los pacientes que aceptaron intervenir en el mismo y a las instituciones pertenecientes al Servicio Canario de la Salud donde se ha desarrollado el estudio (Gerencia de Atención Primaria de Gran Canaria y Hospital Dr. Negrín de Gran Canaria) por facilitar la realización de la investigación.

\section{Conflicto de intereses}

Los autores declaran no tener conflicto de intereses.

\section{BIBLIOGRAFÍA}

1. Salem ZC, Pérez PJ, Henning LE, Uherek PF, Schultz OC, Butte $\mathrm{BJ}$, et al. Heridas. Conceptos generales. Cuadernos de Cirugía. 2018;14(1):90-9. doi:10.4206/cuad.cir.2000.v14n1-15

2. Valencia Basto C. Cicatrización: proceso de reparación tisular. Aproximaciones terapéuticas. Investigaciones Andina. 2010;12(20):85-98.

3. Martín-Paredero V, Arrébola-López M, Berga-Fauria C, PañeIla-Agustí F. Gómez-Moya B. Impacto en la calidad de vida. Angiologia. 2003;55(1):250-9

4. Urzúa MA. Calidad de vida relacionada con la salud: Elementos conceptuales. Rev Med Chilena. 2018:138(3):35865.

5. Melguizo-Herrera E, Díaz-Gómez A, Osorio-Lambi M. Calidad de vida de persona con heridas complejas en Cartagena, Colombia. Rev Salud Pública. 2011:13(6):942-52

6. González de la Torre H, Quintana-Lorenzo ML, Pérez EP, Verdú J. Correlation between health-related quality of life and venous leg ulcer's severity and characteristics: a cross-sectional study. Int Wound J. 2017:14(2):360-8.

7. Santos VLCG, Oliveira AS, Amaral AFS, Nishi ET, Junqueira JB, Kim SHP. Quality of life in patients with chronic wounds: magnitude of changes and predictive factors. Rev Esc Enferm USP. 2017:51:e03250.

8. Consuegra RV, Verdú J. Calidad de vida relacionada con heridas crónicas. Gerokomos. 2010;21(3):131-9.

9. Gale SS, Lurie F, Treadwell T, Vazquez J, Carman T, Partsch H et al. Dominate-Wound. Wounds. 2014;26(1):1-12.

10. Restrepo JC, Verdú J. Instrumentos de monitorización clínica y medida de la cicatrización en úlceras por presión (UPP) y úlceras de la extremidad inferior (UEI). Desarrollo y validación de un índice de medida. Universidad de Alicante, Departamento de Enfermería Comunitaria, Medicina Preventiva, Salud Pública e Historia de la ciencia; 2010

11. Price P, Harding K. Cardiff Wound Impact Schedule: the development of a condition-specific questionnaire to assess health-related quality of life in patients with chronic wounds of the lower limb. Int Wound J. 2004;1:10-7.

12. Lozano-Platonoff A, Contreras-Ruiz J, Dominguez-Cherit J, Cardenas-Sanchez A, Alvarez-Rivero V, Martínez-Regalado JA. Translation, cross-cultural adaptation and validation of the "Cardiff wound impact schedule," a wound-specific quality of life instrument, to the native Spanish of Mexican patients. Int Wound J. 2020:L17(1):167-73. doi: 10.1111/iwj.13253. Epub 2019 Nov 11.

13. Martínez D. Calidad de vida en pacientes con úlcera de pie diabético. Adaptación transcultural al español y validación de los cuestionarios CWIS y DSF-SF. Tesis: (Pendiente de defensa). Universidad de Lleida.

14. Pannier-Fischer F, Rabe E. Epidemiology of chronic venous diseases. Hautarzt. 2003:54(11):1037-44.

15. Finlayson $\mathrm{K}, \mathrm{Wu} \mathrm{M}$, Edwards $\mathrm{HE}$. Identifying risk factors and protective factors for venous leg ulcer recurrence using a theoretical approach: A longitudinal study. Int J Nurs Stud. 2015;52(6):1042-51.

16. Labropoulos N, Wang E, Lanier S, Khan S. Factors Associated with Poor Healing and Recurrence of Venous. Plast Reconstr Surg. 2012;129(1):179-86
17. Marques de Souza E, Bonetti Yoshida W, Aragao de Melo V, Bitencurt de Oliveir L. Ulcer Due to Chronic Venous Disease: A Sociodemographic Study in Northeastern Brazil. Ann Vasc Surg. 2013:27:571-6.

18. Baptista de Oliveira B, de Almeida Nogueira G, Rezende de Carvalho M, Matos de Abreu A. Caracterização dos pacientes com úlcera venosa acompanhados no Ambulatório de Reparo de Feridas. Rev Eletr Enf. 2012:14(1):156-63.

19. Marinello Roura J, Verdú J, coord. Conferencia nacional de consenso sobre las úlceras de la extremidad inferior (C.O.N.U.E.I.). Documento de consenso. 2018. 2. a ed. Madrid Ergon; 2018

20. Van Hecke A, Grypdonck M, Beele H, Vanderwee K, Defloor T. Adherence to leg ulcer lifestyle advice: qualitative and quantitative outcomes associated with a nurse-led intervention. J Clin Nurs. 2011;20:429-43

21. Batista Oliveira, S. et al. Prevalência de úlceras venosas e fatores a ssociados entre adultos de um centro de saúde de Vitória da Conquista - BA. Rev Fund Care Online. 2015 julio-septiembre;7(3):2659-2.

22. Grynberg E, Senet P. Úlceras de la pierna. EMC - Tratado de Medicina. 2015;19(2):1-10.

23. Zimny S, Schatz H, Phohl M. Determinant and estimation of healing times in diabetic foot ulcers. J Diabetes Complications. 2002:16(5):327-32.

24. Montequín JIF. Heridas de difícil cicatrización. La Habana. Cuba: Instituto de Angiología y Cirugía Vascular; 2011. 
25. Sriyani $\mathrm{K}$, et al. Assessment of quality of life of patients with diabetic ulcers by cardiff wound impact schedule (cwis). En: 2nd International Conference of Multidisciplinary Approaches (iCMA), 2015.

26. Kapp S, Santamaría N. The financial and quality-of-life cost to patients living with a chronic wound in the community. Int Wound J. 2017;14(6):1108-19

27. Costa de Oliveira A, De Macêdo Rocha D, Gonçalves Bezerra SM, Leite Rangel Andrare EM, Ribeiro dos Santos AM, Tolstenko Nogueira L. Qualidade de vida de pessoas com feridas crônicas. Acta paul. enferm. 2019;32(2)

28. Jaksa PJ, Mahoney JL. Calidad de vida en pacientes con úlce- ras del pie diabético: validación del Programa de Impacto de Heridas de Cardiff en una población canadiense. Int Wound J. 2010;7(6):502-7.

29. Smith JJ, Guest MG, Greenjalgh RM, Davies AH. Measuring the quality of life in patients with venous ulcers. J Vasc Surg. 2000;(31):642-9.

30. Ribu L, Birkeland K, Hanestad B, Moum T, Rustoen T. A longitudinal study of patients with diabetes and foot ulcers and their health-related quality of life: wound healing and quality of life changes. J Diabetes Complications. 2008;22:400-7.

31. Silva Lucas L Da, Trevisan Martins J, Carmo Cruz Robazzi MLD.
Qualidade de vida dos portadores de ferida em membros inferiores-úlcera de perna. Cienc. enferm. 2008:14(1):43-52.

32. González-Consuegra R, Verdú Soriano J. Calidad de vida y cicatrización en pacientes con úlceras de etiología venosa. Validación del Charing Cross Venous Ulcer Questionnaire, versión española (CCVUQ-e) y del Pressure Ulcer Scale for Healing, versión española (PUSH-e). Resultados preliminares. Gerokomos. 2011;22(3):131-6.

33. Saraiva DMRF, Bandarra AJF, Agostinho EdS, Pereira NMM, Lopes TS. Calidad de vida en pacientes con úlceras venosas crónicas. Rev. Enf. Ref. 2013;3(10):109-18. 